\title{
Stabilization of discrete-time LTI positive systems
}

\author{
DUŠAN KROKAVEC and ANNA FILASOVÁ
}

\begin{abstract}
The paper mitigates the existing conditions reported in the previous literature for control design of discrete-time linear positive systems. Incorporating an associated structure of linear matrix inequalities, combined with the Lyapunov inequality guaranteing asymptotic stability of discrete-time positive system structures, new conditions are presented with which the statefeedback controllers and the system state observers can be designed. Associated solutions of the proposed design conditions are illustrated by numerical illustrative examples.
\end{abstract}

Key words: state feedback stabilization, positive discrete-time observers, linear discretetime positive systems, linear matrix inequalities, asymptotic stability.

\section{Introduction}

Positive systems are often found in the modeling and control of engineering and industrial processes, whose state variables represent quantities that do not have meaning unless they are nonnegative [1]. The mathematical theory of Metzler matrices has a close relationship to the theory of positive linear time-invariant (LTI) dynamical systems, since in the state-space description form the system dynamics matrix of a positive systems is Metzler and the system input and output matrices are nonnegative matrices. Other references can find, e.g., in [2], [3], [4], [5].

The problem of Metzlerian system stabilization and observer design has been previously studied, especially for single input and single output (SISO) continuous-time linear systems, as well as discrete-time linear systems, which have minimal degree of freedom to ensure that a solution exists (see [6], [7], [8], [9] and the references therein). Applicable methods for stabilization of positive linear discrete-time systems, maintaining its positivity when using linear state feedback, are given in [10], [11], [12].

The synthesis problem of state-feedback controllers, guaranteeing the closed-loop system to be asymptotically stable and positive has been investigated by a linear matrix

The authors are with Technical University of Košice, Faculty of Electrical Engineering and Informatics, Department of Cybernetics and Artificial Intelligence, Letná 9, 04200 Košice, Slovakia, e-mails: dusan.krokavec@tuke.sk, anna.filasova@tuke.sk

The work presented in this paper was supported by VEGA, the Grant Agency of the Ministry of Education and the Academy of Science of Slovak Republic, under Grant No. 1/0608/17. This support is very gratefully acknowledged.

Received 11.08.2017. 
inequality (LMI) and the linear programming approach in [13], [14], but as far as the authors know, there is no literature on design of controllers and observers for positive continuous-time or discrete-time linear systems, in which the design conditions are built only on LMIs. The main motivation issue of this paper is to reformulate design conditions for stabilization of linear positive discrete-time systems by using a state-feedback, as well as the conditions for state observers design in the same system structures, by using LMIs. Considering the stable strictly positive matrix structure, algebraic constraints implying from linear programming approach are reformulated as a set of LMIs, which is extended by an LMI, reflecting the Lyapunov stability condition.

The paper is organized as follows. Within the frame of preliminaries, the standard declaration for discrete-time positive systems is presented in Sec. 2 A newly introduced set of LMIs, describing the design conditions for strictly positive SISO discrete-time linear systems, is theoretically substantiated in Sec. 3 and, subsequently, the design conditions for strictly positive multi input, multi-output (MIMO) discrete-time systems are generalized in Sec. 4 An example is provided to demonstrate the proposed approach in Sec. 5, while Sec. 6 draws some conclusions.

Used notations are conventional so that $\boldsymbol{x}^{T}, \boldsymbol{X}^{T}$ denotes transpose of the vector $\boldsymbol{x}$ and matrix $\boldsymbol{X}$, respectively, $\boldsymbol{x}_{+}, \boldsymbol{X}_{+}$indicates a nonnegative vector and a nonnegative matrix, $\boldsymbol{X}=\boldsymbol{X}^{T} \succ 0$ means that $\boldsymbol{X}$ is a symmetric positive definite matrix, $\rho(\boldsymbol{X})$ reports the eigenvalue spectrum of the square matrix $\boldsymbol{X}$, the symbol $\boldsymbol{I}_{n}$ marks the $n$-th order unit matrix, $\operatorname{diag}[\cdot]$ enters up a diagonal matrix, $\mathfrak{R}^{n}, \mathfrak{R}^{n \times r}$ refers to the set of all $n$ dimensional real vectors and $n \times r$ real matrices, respectively, $\mathfrak{R}_{n}^{n}, \mathfrak{R}_{+}^{n \times r}$ signifies the set of all $n$-dimensional real non-negative vectors and $n \times r$ real non-negative matrices, respectively, and $z_{+}$is the set of all positive integers.

\section{System description}

In the paper is considered the discrete-time positive linear dynamical systems, which state-space description is

$$
\begin{gathered}
\boldsymbol{q}(i+1)=\boldsymbol{F} \boldsymbol{q}(i)+\boldsymbol{G} \boldsymbol{u}(i), \\
\boldsymbol{y}(i)=\boldsymbol{C} \boldsymbol{q}(i),
\end{gathered}
$$

where $\boldsymbol{q}(i) \in \mathfrak{R}_{+}^{n}, \boldsymbol{u}(i) \in \mathfrak{R}_{+}^{r}, \boldsymbol{y}(i) \in \mathfrak{R}_{+}^{m}$ stands for state, control input and measurable output, respectively, $\boldsymbol{F} \in \mathfrak{R}_{+}^{n \times n}, \boldsymbol{G} \in \mathfrak{R}_{+}^{n \times r}, \boldsymbol{C} \in \mathfrak{R}_{+}^{m \times n}$ and $i \in Z_{+}$.

Definition 7 [15] (positive linear system) The linear system (1), (2) is said to be positive if and only if for every nonnegative initial state and for every nonnegative input its state and output are nonnegative.

Definition 8 [16] A matrix $\boldsymbol{X} \in \mathfrak{R}_{+}^{p \times q}$ and a vector $\boldsymbol{x} \in \mathfrak{R}_{+}^{p}$ are said to be nonnegative if all its entries are nonnegative and at least one is positive. 
Definition 9 [17] A square matrix $\boldsymbol{F} \in \mathfrak{R}_{+}^{n \times n}$ is positive if its elements are nonnegative. A square matrix $\boldsymbol{F} \in \mathfrak{R}_{+}^{n \times n}$ is stable strictly positive matrix if is Schur and all its elements are positive.

Remark 3 Considering a strictly Metzlerian continuous-time systems, then all diagonal elements of the matrix $\boldsymbol{A}$ are negative, all off-diagonal elements of $\boldsymbol{A}$ are positive and the matrix $\boldsymbol{B}$ is nonnegative [18]. Taking this continuous system pair $(\boldsymbol{A}, \boldsymbol{B})$, the associated discrete-time system pair is [19]

$$
\begin{gathered}
\boldsymbol{F}=\boldsymbol{I}_{n}+\boldsymbol{A} \frac{t_{s}}{1 !}+\boldsymbol{A}^{2} \frac{t_{s}^{2}}{2 !}+\cdots+\boldsymbol{A}^{n} \frac{t_{s}^{n}}{n !}+\cdots, \\
\boldsymbol{G}=\left(\boldsymbol{I}_{n} \frac{t_{s}}{1 !}+\boldsymbol{A} \frac{t_{s}^{2}}{2 !}+\cdots+\boldsymbol{A}^{n-1} \frac{t_{s}^{n}}{n !}+\cdots\right) \boldsymbol{B},
\end{gathered}
$$

where $t_{s}$ is the sampling period. Obviously, for a sufficiently small sampling period, all elements of $\boldsymbol{F}$ are positive if $\boldsymbol{A}$ is strictly Metzler matrix and $\boldsymbol{G}$ is nonnegative if $\boldsymbol{B}$ is nonnegative.

Proposition 1 [20] A positive matrix $\boldsymbol{F}$ is stable if and only if is diagonally dominant.

Proposition 2 [21] A solution $\boldsymbol{q}(i)$ of (1) is asymptotically stable and positive, i.e., $\lim _{i \rightarrow \infty} \boldsymbol{q}(i)=\mathbf{0}$ while $\boldsymbol{q}(i) \in \mathfrak{R}_{+}^{n}$ for $\boldsymbol{u}(i) \in \mathfrak{R}_{+}^{r}$ and the initial value $\boldsymbol{q}(0) \in \mathfrak{R}_{+}^{n}$, if $\boldsymbol{F}$ is a stable positive matrix and $\boldsymbol{G} \in \mathfrak{R}_{+}^{n \times r}$ is a non-negative matrix. The linear system (1), (2) is asymptotically stable and positive if $\boldsymbol{F}$ is a stable positive matrix, $\boldsymbol{G} \in \mathfrak{R}_{+}^{n \times r}$, $\boldsymbol{C} \in \mathfrak{R}_{+}^{m \times n}$ are non-negative matrices and $\boldsymbol{y}(i) \in \mathfrak{R}_{+}^{m}$ for $\boldsymbol{u}(i) \in \mathfrak{R}_{+}^{r}$ and the initial value $\boldsymbol{q}(0) \in \mathfrak{R}_{+}$. The linear system (1), (2) is asymptotically stable and internally positive if $\boldsymbol{F}$ is a stable positive matrix and $\boldsymbol{G} \in \mathfrak{R}_{+}^{n \times r}, \boldsymbol{C} \in \mathfrak{R}_{+}^{m \times n}$ are non-negative matrices.

Proposition 3 [22] (Lyapunov inequalities) Autonomous system (1) is asymptotically stable if there exist symmetric positive definite matrices $\boldsymbol{P}, \boldsymbol{Q} \in \mathfrak{R}^{n \times n}$ or $\boldsymbol{V}, \boldsymbol{U} \in \mathfrak{R}^{n \times n}$ such that

$$
\begin{gathered}
\boldsymbol{P}=\boldsymbol{P}^{T} \succ 0, \quad \boldsymbol{Q}=\boldsymbol{Q}^{T} \succ 0, \quad\left[\begin{array}{cc}
-\boldsymbol{P}+\boldsymbol{Q} & \boldsymbol{P} \boldsymbol{F}^{T} \\
\boldsymbol{F} \boldsymbol{P} & -\boldsymbol{P}
\end{array}\right] \prec 0, \\
\boldsymbol{V}=\boldsymbol{V}^{T} \succ 0, \quad \boldsymbol{U}=\boldsymbol{U}^{T} \succ 0, \quad\left[\begin{array}{cc}
-\boldsymbol{V}+\boldsymbol{U} & \boldsymbol{F}^{T} \boldsymbol{V} \\
\boldsymbol{V} \boldsymbol{F} & -\boldsymbol{V}
\end{array}\right] \prec 0 .
\end{gathered}
$$

Proposition 4 [23] If $\boldsymbol{X} \in \mathfrak{R}^{n \times n}$ is a symmetric positive definite matrix it yields the following relation

$$
\boldsymbol{X}=\boldsymbol{X}^{T} \succ 0 \Leftrightarrow \frac{1}{2} \boldsymbol{X}+\frac{1}{2} \boldsymbol{X}^{T} \succ 0 .
$$


Definition 10 [24] (congruent modulo $n$ ) Let $n$ be a fixed positive integer. Two integers $j$ and $h$ are congruent modulo $n$ if they differ by an integral multiple of the integer $n$ (they leave the same remainder when divided by $n$ ). If $j$ and $h$ are congruent modulo $n$, the expression $(j=h)_{\bmod n}$ is called a congruence, and the number $n$ is called the modulus of the congruence.

The statement $(j=h)_{\bmod n}$ is equivalent to the statement " $(j-h)$ is divisible by $n$ " or to the statement "there is an integer $m$ for which $j-h=m n$ " and the word "modulo" generally means "to the modulus".

Definition 11 [25] Let $S=\{0,1,2, \ldots, n-1\}$ be the complete set of residues for any positive integer $n$. The addition modulo $n$ on $S$ is $(j+h)_{\bmod n}=r$, where $r$ is the element of $S$ to which the result of the usual sum of integers $j$ and $h$ is congruent modulo $n$.

Corollary 1 The problem of indexing in this paper is that the rows and columns of a square matrix of dimension $n \times n$ are generally denoted from 1 to $n$ and not from 0 to $n-1$. From this reason let $S=\{0,1,2, \ldots, n\}$ be the complete set of residues for any positive integer $n+1$. Then, the addition modulo $n+1$ on $S$ is in the following defined as $(j+h)_{\bmod n+1}=r+1$, where $r$ is the element of $S$ to which the result of the usual sum of integers $j$ and $k$ is congruent modulo $n+1$. The used shorthand symbolical notation for $(j+h)_{\bmod n+1}=r+1$ is so $(j+h)_{(1 \leftrightarrow n) / n}=r+1$.

\section{Strictly positive SISO systems}

The systems under consideration in this section are linear discrete-time SISO dynamical systems represented in state-space form as

$$
\begin{gathered}
\boldsymbol{q}(i+1)=\boldsymbol{F} \boldsymbol{q}(i)+\boldsymbol{g} u(i), \\
y(i)=\boldsymbol{c}^{T} \boldsymbol{q}(i),
\end{gathered}
$$

where $\boldsymbol{q}(i) \in \mathfrak{R}_{+}^{n}$ is the vector of the system state variables, $u(i) \in \mathfrak{R}_{+}, y(i) \in \mathfrak{R}_{+}$are the input and output variables, $\boldsymbol{F} \in \mathfrak{R}_{+}^{n \times n}$ is a strictly positive matrix, $\boldsymbol{g} \in \mathfrak{R}_{+}^{n}, \boldsymbol{c} \in \mathfrak{R}_{c}^{n}$ are non-negative vectors, the pair $(\boldsymbol{F}, \boldsymbol{g})$ is controllable an the pair $\left(\boldsymbol{F}, \boldsymbol{c}^{T}\right)$ is observable.

Considering the system (8), (9), the full state control law is defined as

$$
\boldsymbol{u}(i)=-\boldsymbol{k}^{T} \boldsymbol{q}(i),
$$

where $\boldsymbol{k} \in \mathfrak{R}_{+}^{n}$ is the control law gain vector. Thus, using (8), (10), it yields

$$
\begin{gathered}
\boldsymbol{q}(i)=\left(\boldsymbol{F}-\boldsymbol{g} \boldsymbol{k}^{T}\right) \boldsymbol{q}(i)=\boldsymbol{F}_{c} \boldsymbol{q}(i), \\
y(i)=\boldsymbol{c}^{T} \boldsymbol{q}(i),
\end{gathered}
$$


where

$$
\boldsymbol{F}_{c}=\boldsymbol{F}-\boldsymbol{g} \boldsymbol{k}^{T}
$$

is the closed-loop system dynamics matrix.

Considering the observable system (8), (9), the Luenberger observer is given as

$$
\begin{gathered}
\dot{\boldsymbol{q}}_{e}(t)=\boldsymbol{A} \boldsymbol{q}_{e}(t)+\boldsymbol{b} \boldsymbol{u}(t)+\boldsymbol{j}\left(y(t)-y_{e}(t)\right), \\
y_{e}(t)=\boldsymbol{c}^{T} \boldsymbol{q}_{e}(t),
\end{gathered}
$$

where $\boldsymbol{j} \in \mathfrak{R}_{+}^{n}$ is the observer gain vector. Using (8), (9) and (14), (15) it yields

$$
\dot{\boldsymbol{e}}(t)=\left(\boldsymbol{F}-\boldsymbol{j} \boldsymbol{c}^{T}\right) \boldsymbol{e}(t)=\boldsymbol{F}_{e} \boldsymbol{e}(t),
$$

where

$$
\boldsymbol{e}(t)=\boldsymbol{q}(t)-\boldsymbol{q}_{e}(t), \quad \boldsymbol{F}_{e}=\boldsymbol{F}-\boldsymbol{j} \boldsymbol{c}^{T},
$$

$\boldsymbol{q}_{e}(t) \in \mathfrak{R}_{+}^{n}$ is the vector of the system state estimate and $y_{e}(t) \in \mathfrak{R}_{+}$the output variable estimate.

Note, the controller has to be designed not only to stabilize the system, but also to render the closed-loop system matrix $\boldsymbol{F}_{c}$ strictly positive and Schur. In the same way, the observer has to be designed so that the observer system matrix $\boldsymbol{F}_{e}$ is strictly positive and Schur.

Theorem 3 The closed-loop system (11), (12) is stable strictly positive if the system (8), (9) is strictly positive and there exists positive definite diagonal matrices $\boldsymbol{P}, \boldsymbol{Q}, \boldsymbol{R} \in \mathfrak{R}^{n \times n}$ such that for $h=0,1,2, \ldots n-1$

$$
\begin{aligned}
& {\left[\begin{array}{cc}
-\boldsymbol{P}+\boldsymbol{Q} & * \\
\boldsymbol{F P}-\boldsymbol{g} \boldsymbol{r}^{T} & -\boldsymbol{P}
\end{array}\right] \prec 0} \\
& \frac{1}{2}\left(\boldsymbol{T}^{h} \boldsymbol{F}(j, j+h)_{(1 \leftrightarrow n) / n} \boldsymbol{T}^{h T} \boldsymbol{P}-\boldsymbol{T}^{h} \boldsymbol{G}_{d} \boldsymbol{T}^{h T} \boldsymbol{R}\right)+(*) \succ 0, \\
& \boldsymbol{P}=\operatorname{diag}\left[\begin{array}{llll}
p_{1} & p_{2} & \cdots & p_{n}
\end{array}\right] \succ 0, \\
& \boldsymbol{Q}=\operatorname{diag}\left[\begin{array}{llll}
q_{1} & q_{2} & \cdots & q_{n}
\end{array}\right] \succ 0, \\
& \boldsymbol{R}=\operatorname{diag}\left[\begin{array}{llll}
r_{1} & r_{2} & \cdots & r_{n}
\end{array}\right] \succ 0, \\
& \boldsymbol{r}^{T}=\left[\begin{array}{llll}
r_{1} & r_{2} & \cdots & r_{n}
\end{array}\right]=\boldsymbol{l}^{T} \boldsymbol{R}, \quad \boldsymbol{l}=\left[\begin{array}{llll}
1 & 1 & \cdots & 1
\end{array}\right]^{T},
\end{aligned}
$$

where

$$
\boldsymbol{T}=\left[\begin{array}{ccccc}
0 & 0 & \cdots & 0 & 1 \\
1 & 0 & \cdots & 0 & 0 \\
& & \ddots & & \\
0 & 0 & \cdots & 1 & 0
\end{array}\right], \quad \boldsymbol{T}^{-1}=\boldsymbol{T}^{T}
$$




$$
\begin{aligned}
& \boldsymbol{g}^{T}=\left[\begin{array}{llll}
g_{1} & g_{2} & \cdots & g_{n}
\end{array}\right], \\
& \boldsymbol{G}_{d}=\operatorname{diag}\left[\begin{array}{llll}
g_{1} & g_{2} & \cdots & g_{n}
\end{array}\right]=\operatorname{diag}\left[\left\{g_{j}\right\}_{j=1, \ldots, n}\right], \\
& \boldsymbol{F}(j, j+h)_{(1 \leftrightarrow n) / n}=\operatorname{diag}\left[\begin{array}{lllllll}
f_{1,1+h} & f_{2,2+h} & \cdots & f_{n-h, n} & f_{n-h+1,1} & \cdots & f_{n, h}
\end{array}\right],
\end{aligned}
$$

while $\boldsymbol{F}(j, j+h)_{(1 \leftrightarrow n) / n}, \boldsymbol{T}, \boldsymbol{G}_{d} \in \mathfrak{R}_{+}^{n \times n}$.

When the above conditions hold, the control law gain vector $\boldsymbol{k}^{T}$ is given as

$$
\boldsymbol{K}_{d}=\boldsymbol{R} \boldsymbol{P}^{-1}, \quad \boldsymbol{k}^{T}=\boldsymbol{l}^{T} \boldsymbol{K}_{d},
$$

where $\boldsymbol{K}_{d} \in \mathfrak{R}_{+}^{n \times n}$.

Hereafter, $*$ denotes the symmetric item in a symmetric matrix.

Proof Writing the closed-loop system matrix $\boldsymbol{F}_{c}$ as follows

$$
\left[\begin{array}{cccc}
f_{11} & f_{12} & \cdots & f_{1 n} \\
f_{21} & f_{22} & \cdots & f_{2 n} \\
& & \ddots & \\
f_{n 1} & f_{n 2} & \cdots & f_{n n}
\end{array}\right]-\left[\begin{array}{c}
g_{1} \\
g_{2} \\
\vdots \\
g_{n}
\end{array}\right]\left[\begin{array}{llll}
k_{1} & k_{2} & \cdots & k_{n}
\end{array}\right] \prec 0,
$$

it is evident that $\boldsymbol{F}_{c}$ be a strictly positive matrix if all its elements are positive, i.e.,

$$
\begin{gathered}
f_{j j}-g_{j} k_{j}>0 \text { for all } j=1,2, \ldots, n \\
f_{j l}-g_{j} k_{l}>0 \text { for all } j, l=1,2, \ldots, n, j \neq l .
\end{gathered}
$$

To solve by an LMI solver, LMIs have to be symmetric and so, using the notations (26), (27), then (30) can be rewritten in the diagonal matrix structure

$$
\boldsymbol{F}(j, j)_{(1 \leftrightarrow n) / n}-\boldsymbol{G}_{d} \boldsymbol{K}_{d} \succ 0,
$$

where $\boldsymbol{K}_{d}=\operatorname{diag}\left[\left\{k_{j}\right\}_{j=1, \ldots, n}\right]$ is a diagonal matrix variable.

Rewriting (29) as

$$
\left[\begin{array}{ccccc}
f_{12} & f_{13} & \cdots & f_{1 n} & f_{11} \\
f_{22} & f_{23} & \cdots & f_{2 n} & f_{21} \\
& & \vdots & & \\
f_{n 2} & f_{n 3} & \cdots & f_{n n} & f_{n 1}
\end{array}\right]-\left[\begin{array}{c}
g_{1} \\
g_{2} \\
\vdots \\
g_{n}
\end{array}\right]\left[\begin{array}{lllll}
k_{2} & k_{3} & \cdots & k_{n} & k_{1}
\end{array}\right],
$$

it can set for the diagonal elements of (33)

$$
\boldsymbol{F}(j, j+1)_{(1 \leftrightarrow n) / n}-\boldsymbol{G}_{d} \boldsymbol{K}_{d c 1} \succ 0,
$$

where $\boldsymbol{K}_{d c 1}$ is the diagonal matrix $\boldsymbol{K}_{d}$ with one circular shift of its diagonal elements. 
Repeating this procedure $h$-times, it can be obtained from (29) that

$$
\left[\begin{array}{cccccc}
f_{1,1+h} & \cdots & f_{1, n} & f_{1,1} & \cdots & f_{1, h} \\
f_{2, i+h} & \cdots & f_{2, n} & f_{2,1} & \cdots & f_{2, h} \\
\vdots & \ddots & \vdots & \vdots & \ddots & \vdots \\
f_{n, i+h} & \cdots & f_{n, n} & f_{n, 1} & \cdots & f_{n, h}
\end{array}\right]-\left[\begin{array}{c}
g_{1} \\
g_{2} \\
\vdots \\
g_{n}
\end{array}\right]\left[\begin{array}{llll}
k_{1+h} & k_{2+h} & \cdots & k_{h}
\end{array}\right]
$$

and so, consequently,

$$
\boldsymbol{F}(j, j+h)_{(1 \leftrightarrow n) / n}-\boldsymbol{G}_{d} \boldsymbol{K}_{d c h} \succ 0,
$$

where $\boldsymbol{K}_{d c h}$ is the diagonal matrix $\boldsymbol{K}_{d}$ with $h$ circular shifts of its diagonal elements.

Using the permutation matrix $\boldsymbol{T}$ [26] of the structure (24), it can be easily verified that for $h=0,1,2, \ldots n-1$ yields the following realation

$$
\boldsymbol{K}_{d}=\boldsymbol{T}^{h} \boldsymbol{K}_{d c h} \boldsymbol{T}^{-h}=\boldsymbol{T}^{h} \boldsymbol{K}_{d c h} \boldsymbol{T}^{h T} .
$$

Thus, pre-multiplying the right side by $\boldsymbol{T}^{h T}$ and post-multiplying the left side by $\boldsymbol{T}^{h}$ then (36) leads to

$$
\begin{gathered}
\boldsymbol{T}^{h} \boldsymbol{F}(j, j+h)_{(1 \leftrightarrow n) / n} \boldsymbol{T}^{h T}-\boldsymbol{T}^{h} \boldsymbol{G}_{d} \boldsymbol{T}^{h T} \boldsymbol{T}^{h} \boldsymbol{K}_{d c h} \boldsymbol{T}^{h T}= \\
=\boldsymbol{T}^{h} \boldsymbol{F}(j, j+h)_{(1 \leftrightarrow n) / n} \boldsymbol{T}^{h T}-\boldsymbol{T}^{h} \boldsymbol{G}_{d} \boldsymbol{T}^{h T} \boldsymbol{K}_{d} \succ 0
\end{gathered}
$$

and, since (38) is a symmetric matrix, using (7), then (38) can be rewritten as

$$
\frac{1}{2}\left(\boldsymbol{T}^{h} \boldsymbol{F}(j, j+h)_{(1 \leftrightarrow n) / n} \boldsymbol{T}^{h T}-\boldsymbol{T}^{h} \boldsymbol{G}_{d} \boldsymbol{T}^{h T} \boldsymbol{K}_{d}\right)+(*) \succ 0,
$$

Multiplying the right side of (39) by a positive definite diagonal matrix $\boldsymbol{P} \in \mathfrak{R}_{+}^{n \times n}$ gives

$$
\frac{1}{2}\left(\boldsymbol{T}^{h} \boldsymbol{A}(j, j+h)_{(1 \leftrightarrow n) / n} \boldsymbol{T}^{h T} \boldsymbol{P}-\boldsymbol{T}^{h} \boldsymbol{B}_{d} \boldsymbol{T}^{h T} \boldsymbol{K}_{d} \boldsymbol{P}\right)+(*) \succ 0
$$

and with the notation

$$
\boldsymbol{R}=\boldsymbol{K}_{d} \boldsymbol{P}
$$

then (39) implies (19).

Inserting the closed-loop system matrix (13) into (5) means

$$
\left[\begin{array}{cc}
-\boldsymbol{P}+\boldsymbol{Q} & \boldsymbol{P}\left(\boldsymbol{F}-\boldsymbol{g} \boldsymbol{k}^{T}\right)^{T} \\
\left(\boldsymbol{F}-\boldsymbol{g} \boldsymbol{k}^{T}\right) \boldsymbol{P} & -\boldsymbol{P}
\end{array}\right] \prec 0
$$

and since (41) gives

$$
\boldsymbol{r}^{T}=\boldsymbol{k}^{T} \boldsymbol{P}
$$

then (42) implies (18). This concludes proof. 
Remark 4 It can be noted, conditions (18)-(22) are all LMIs that is, they are convex in the defined matrix variables. Moreover, the necessary diagonal matrix variable structure of $\boldsymbol{K}_{c}$ directly implies the diagonal matrix variable strictures of $\boldsymbol{P}, \boldsymbol{R}$ in Theorem 3. Since, in the terms of the Krasovskii theorem [27], the matrices $\boldsymbol{Q}, \boldsymbol{U}$ in Proposition 3 can be zero matrices, or a symmetric positive definite matrices, this way can be also applied in (18), as well as in the inequalities exploited this proposition properties in the following parts of the paper.

To simplify obtaining relation in $\boldsymbol{A}(j, j+h)_{(1 \leftrightarrow n) / n}$ it is possible to construct the following matrix

$$
\boldsymbol{F}^{\circ}=\left[\begin{array}{ll}
\boldsymbol{F} & \boldsymbol{F}
\end{array}\right]=\left[\begin{array}{cccccccc}
f_{11} & f_{12} & \cdots & f_{1 n} & f_{11} & f_{12} & \cdots & f_{2 n} \\
\vdots & & \ddots & & \vdots & & \ddots & \\
f_{11} & f_{12} & \cdots & f_{1 n} & f_{11} & f_{12} & \cdots & f_{1 n}
\end{array}\right] .
$$

Then, using the main diagonal elements and the set of $n-1$ upper sub-diagonals of dimension of $n$, the matrices $\boldsymbol{A}(j, j+h)_{(1 \leftrightarrow n) / n}$ can be sequentially constructed for $h=$ $0,1,2, \ldots n-1$ from (44).

Theorem 4 The Luenberger observer (14), (15) is stable strictly positive if the system (8), (9) is strictly positive and there exists a positive definite diagonal matrices $\boldsymbol{U}, \boldsymbol{V}, \boldsymbol{W} \in$ $\mathfrak{R}^{n \times n}$ such that for $h=0,1,2, \ldots n-1$

$$
\begin{gathered}
{\left[\begin{array}{cc}
-\boldsymbol{V}+\boldsymbol{U} & \boldsymbol{F}^{T} \boldsymbol{V}-\boldsymbol{c} \boldsymbol{w}^{T} \\
* & -\boldsymbol{V}
\end{array}\right] \prec 0,} \\
\frac{1}{2}\left(\boldsymbol{V} \boldsymbol{T}^{h} \boldsymbol{F}(j+h, j)_{(1 \leftrightarrow n) / n} \boldsymbol{T}^{h T}-\boldsymbol{W} \boldsymbol{T}^{h} \boldsymbol{C}_{d} \boldsymbol{T}^{h T}\right)+(*) \succ 0, \\
\boldsymbol{V}=\operatorname{diag}\left[\begin{array}{llll}
v_{1} & v_{2} & \cdots & v_{n}
\end{array}\right] \succ 0, \\
\boldsymbol{W}=\operatorname{diag}\left[\begin{array}{llll}
w_{1} & w_{2} & \cdots & w_{n}
\end{array}\right] \succ 0, \\
\boldsymbol{U}=\operatorname{diag}\left[\begin{array}{llll}
u_{1} & u_{2} & \cdots & u_{n}
\end{array}\right] \succ 0, \\
\boldsymbol{w}^{T}=\left[\begin{array}{lllll}
w_{1} & w_{2} & \cdots & w_{n}
\end{array}\right]=\boldsymbol{l}^{T} \boldsymbol{W}, \quad \boldsymbol{l}=\left[\begin{array}{llll}
1 & 1 & \cdots & 1
\end{array}\right]^{T},
\end{gathered}
$$

where $\boldsymbol{T}$ is defined in (21),

$$
\begin{aligned}
& \boldsymbol{c}^{T}=\left[\begin{array}{llll}
c_{1} & c_{2} & \cdots & c_{n}
\end{array}\right], \\
& \boldsymbol{C}_{d}=\operatorname{diag}\left[\begin{array}{llll}
c_{1} & c_{2} & \cdots & c_{n}
\end{array}\right]=\operatorname{diag}\left[\left\{c_{i}\right\}_{i=1, \ldots, n}\right] \text {, }
\end{aligned}
$$

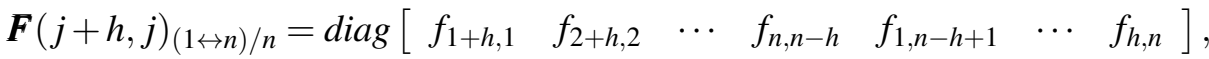

while $\boldsymbol{F}(j+h, j)_{(1 \leftrightarrow n) / n}, \boldsymbol{C}_{d} \in \mathfrak{R}_{+}^{n \times n}$.

When the above conditions hold, the observer gain vector $\boldsymbol{j}$ is given as

$$
\boldsymbol{J}_{d}=\boldsymbol{V}^{-1} \boldsymbol{W}, \quad \boldsymbol{j}=\boldsymbol{J}_{d} \boldsymbol{l}
$$

where $\boldsymbol{J}_{d} \in \mathfrak{R}_{+}^{n \times n}$. 
Proof Since the duality principle can be applied in observer design, while the triple $\left(\boldsymbol{F}^{T}, \boldsymbol{c}, \boldsymbol{g}^{T}\right)$ is dual to the triple $\left(\boldsymbol{F}, \boldsymbol{g}, \boldsymbol{c}^{T}\right)$, the duality has to be applied at first order on the structure of (44).

Because $\boldsymbol{F}_{e}$ be a strictly positive matrix if

$$
\begin{gathered}
f_{j j}-j_{j} c_{j}>0 \text { for all } j=1,2, \ldots, n, \\
a_{j k}-j_{j} c_{k}>0 \text { for all } j, k=1,2, \ldots, n, i \neq j,
\end{gathered}
$$

respecting the duality principle, the condition (55) can be written as (compare with (32))

$$
\boldsymbol{F}(j, j)_{(1 \leftrightarrow n) / n}-\boldsymbol{J}_{d} \boldsymbol{C}_{d} \succ 0,
$$

where $\boldsymbol{J}_{d}=\operatorname{diag}\left[\left\{j_{l}\right\}_{l=1, \ldots, n}\right]$ is the diagonal matrix variable.

Applying the duality principle on (44) it can obtain with respect to (38) the dual form

$$
\boldsymbol{F}(j+h, j)_{(1 \leftrightarrow n) / n}-\boldsymbol{J}_{d c h} \boldsymbol{C}_{d} \succ 0
$$

and using the matrix $\boldsymbol{T}$ of the structure (24) it yields for $h=0,1,2, \ldots n-1$ that

$$
\boldsymbol{J}_{d}=\boldsymbol{T}^{h} \boldsymbol{J}_{d c h} \boldsymbol{T}^{-h}=\boldsymbol{T}^{h} \boldsymbol{J}_{d c h} \boldsymbol{T}^{h T} .
$$

Pre-multiplying the left side by $\boldsymbol{T}^{h}$ and post-multiplying the right side by $\boldsymbol{T}^{h T}$ then the symmetric structure (58) gives

$$
\begin{gathered}
\boldsymbol{T}^{h} \boldsymbol{F}(j+h, j)_{(1 \leftrightarrow n) / n} \boldsymbol{T}^{h T}-\boldsymbol{T}^{h} \boldsymbol{J}_{d c h} \boldsymbol{T}^{h T} \boldsymbol{T}^{h} \boldsymbol{C}_{d} \boldsymbol{T}^{h T}= \\
=\boldsymbol{T}^{h} \boldsymbol{F}(j+h, j)_{(1 \leftrightarrow n) / n} \boldsymbol{T}^{h T}-\boldsymbol{J}_{d} \boldsymbol{T}^{h} \boldsymbol{C}_{d} \boldsymbol{T}^{h T} \succ 0,
\end{gathered}
$$

which means that

$$
\frac{1}{2}\left(\boldsymbol{T}^{h} \boldsymbol{F}(j+h, j)_{(1 \leftrightarrow n) / n} \boldsymbol{T}^{h T}-\boldsymbol{J}_{d} \boldsymbol{T}^{h} \boldsymbol{C}_{d} \boldsymbol{T}^{h T}\right)+(*) \succ 0 .
$$

Therefore, multiplying the left side of (61) by $\boldsymbol{V}$ gives

$$
\frac{1}{2}\left(\boldsymbol{V} \boldsymbol{T}^{h} \boldsymbol{F}(j+h, j)_{(1 \leftrightarrow n) / n} \boldsymbol{T}^{h T}-\boldsymbol{V} \boldsymbol{J}_{d} \boldsymbol{T}^{h} \boldsymbol{C}_{d} \boldsymbol{T}^{h T}\right)+(*) \succ 0
$$

and with the notation

$$
\boldsymbol{W}=\boldsymbol{V} \boldsymbol{J}_{d}
$$

then (62) implies (46).

Inserting the observer system matrix (17) into (6) leads to

$$
\left[\begin{array}{cc}
-\boldsymbol{V}+\boldsymbol{U} & \left(\boldsymbol{F}-\boldsymbol{j} \boldsymbol{c}^{T}\right)^{T} \boldsymbol{V} \\
\boldsymbol{V}\left(\boldsymbol{F}-\boldsymbol{j} \boldsymbol{c}^{T}\right) & -\boldsymbol{V}
\end{array}\right] \prec 0
$$

and since (63) gives

$$
\boldsymbol{w}=\boldsymbol{V} \boldsymbol{j}, \quad \boldsymbol{w}^{T}=\boldsymbol{j}^{T} \boldsymbol{V},
$$


then (64) implies (45). This concludes proof.

Also the conditions (45)-(49) are all LMIs that is, they are convex in the defined matrix variables.

To support the duality principle it is possible to construct the matrix

$$
\boldsymbol{F}^{\diamond}=\left[\begin{array}{c}
\boldsymbol{F} \\
\boldsymbol{F}
\end{array}\right]=\left[\begin{array}{cccc}
f_{11} & f_{12} & \cdots & f_{1 n} \\
f_{21} & f_{22} & \cdots & f_{2 n} \\
\vdots & & \ddots & \\
f_{n 1} & f_{n 2} & \cdots & f_{n n} \\
f_{11} & f_{12} & \cdots & f_{1 n} \\
f_{21} & f_{22} & \cdots & f_{2 n} \\
\vdots & & \ddots & \\
f_{n 1} & f_{n 2} & \cdots & f_{n n}
\end{array}\right]
$$

and exploiting the main diagonal as well as the set of $n-1$ lower sub-diagonals of dimension of $n$ it can construct sequentially the matrices $\boldsymbol{F}(j+h, j)_{(1 \leftrightarrow n) / n}$ for $h=$ $0,1,2, \ldots n-1$ from (66).

\section{Strictly positive MIMO systems}

Linear discrete-time closed-loop MIMO dynamical systems, obtained from the controllable system (1), (2) by using the state control law

$$
\boldsymbol{u}(i)=-\boldsymbol{K} \boldsymbol{q}(i), \quad \boldsymbol{K} \in \mathfrak{R}_{+}^{r \times n},
$$

is described by the state-space equations

$$
\begin{gathered}
\boldsymbol{q}(i+1)=(\boldsymbol{F}-\boldsymbol{G} \boldsymbol{K}) \boldsymbol{q}(i)=\boldsymbol{F}_{c} \boldsymbol{q}(i), \\
\boldsymbol{y}(i)=\boldsymbol{C} \boldsymbol{q}(i),
\end{gathered}
$$

where

$$
\boldsymbol{G}=\left[\begin{array}{lll}
\boldsymbol{g}_{1} & \cdots & \boldsymbol{g}_{r}
\end{array}\right], \quad \boldsymbol{K}^{T}=\left[\begin{array}{lll}
\boldsymbol{k}_{1} & \cdots & \boldsymbol{k}_{r}
\end{array}\right], \quad \boldsymbol{F}_{c}=\boldsymbol{F}-\sum_{k=1}^{r} \boldsymbol{g}_{g} \boldsymbol{k}_{k}^{T} .
$$

In the same manner, the MIMO Luenberger observer, associated with the observable system (1), (2), is given as

$$
\begin{gathered}
\boldsymbol{q}_{e}(i+1)=\boldsymbol{F} \boldsymbol{q}_{e}(i)+\boldsymbol{G} \boldsymbol{u}(i)+\boldsymbol{J}\left(\boldsymbol{y}(i)-\boldsymbol{y}_{e}(i)\right), \\
\boldsymbol{y}_{e}(i)=\boldsymbol{C} \boldsymbol{q}_{e}(i), \\
\boldsymbol{e}(i+1)=(\boldsymbol{F}-\boldsymbol{J} \boldsymbol{C}) \boldsymbol{e}(i)=\boldsymbol{F}_{e} \boldsymbol{e}(i),
\end{gathered}
$$


where

$$
\begin{gathered}
\boldsymbol{e}(i)=\boldsymbol{q}(i)-\boldsymbol{q}_{e}(i), \\
\boldsymbol{C}^{T}=\left[\begin{array}{lll}
\boldsymbol{c}_{1} & \cdots & \boldsymbol{c}_{r}
\end{array}\right], \quad \boldsymbol{F}_{e}=\boldsymbol{F}-\sum_{k=1}^{r} \boldsymbol{j}_{k} \boldsymbol{c}_{k}^{T}, \\
\boldsymbol{J}=\left[\begin{array}{lll}
\boldsymbol{j}_{1} & \cdots & \boldsymbol{j}_{m}
\end{array}\right], \quad \boldsymbol{J} \in \mathfrak{R}_{+}^{n \times m} .
\end{gathered}
$$

Naturally, if $\boldsymbol{F} \in \mathfrak{R}_{+}^{n \times n}$ is a strictly positive matrix, and $\boldsymbol{G} \in \mathfrak{R}_{+}^{n \times r}, \boldsymbol{C} \in \mathfrak{R}_{+}^{m \times n}$ are non-negative matrices, the system (1), (2) is positive system. Thus, it is necessary to render the closed-loop system matrix $\boldsymbol{F}_{c}$ and observer system matrix $\boldsymbol{F}_{e}$ be stable strictly positive matrices.

Theorem 5 The closed-loop system (11), (12) is stable strictly positive if the system (1), (2) is strictly positive and there exists positive definite diagonal matrices $\boldsymbol{P}, \boldsymbol{R}_{k}, \boldsymbol{Q} \in \mathfrak{R}^{n \times n}$ such that for $h=0,1,2, \ldots n-1, k=1,2, \ldots r$,

$$
\begin{gathered}
{\left[\begin{array}{cc}
-\boldsymbol{P}+\boldsymbol{Q} & * \\
\boldsymbol{F} \boldsymbol{P}-\sum_{k=1}^{r} \boldsymbol{g}_{k} \boldsymbol{r}_{k}^{T} & -\boldsymbol{P}
\end{array}\right] \prec 0,} \\
\frac{1}{2}\left(\boldsymbol{T}^{h} \boldsymbol{F}(j, j+h)_{(1 \leftrightarrow n) / n} \boldsymbol{T}^{h T} \boldsymbol{P}-\sum_{k=1}^{r} \boldsymbol{T}^{h} \boldsymbol{G}_{d k} \boldsymbol{T}^{h T} \boldsymbol{R}_{k}\right)+(*) \succ 0, \\
\boldsymbol{P}=\operatorname{diag}\left[\begin{array}{llll}
p_{1} & p_{2} & \cdots & p_{n}
\end{array}\right] \succ 0, \\
\boldsymbol{Q}=\operatorname{diag}\left[\begin{array}{llll}
q_{1} & q_{2} & \cdots & q_{n}
\end{array}\right] \succ 0, \\
\boldsymbol{R}_{k}=\operatorname{diag}\left[\begin{array}{llll}
r_{k 1} & r_{k 2} & \cdots & r_{k n}
\end{array}\right] \succ 0, \\
\boldsymbol{r}_{k}^{T}=\left[\begin{array}{ccccc}
r_{k 1} & r_{k 2} & \cdots & r_{k n}
\end{array}\right]=\boldsymbol{l}^{T} \boldsymbol{R}_{k}, \quad \boldsymbol{l}=\left[\begin{array}{lll}
1 & 1 & \cdots
\end{array}\right]^{T},
\end{gathered}
$$

where $\boldsymbol{T}$ is defined in (25), $\boldsymbol{F}(j, j+1)_{(1 \leftrightarrow n) / n}$ in (27),

$$
\begin{gathered}
\boldsymbol{G}=\left[\begin{array}{llll}
\boldsymbol{g}_{1} & \boldsymbol{g}_{2} & \cdots & \boldsymbol{g}_{r}
\end{array}\right]=\left[\begin{array}{cccc}
g_{11} & g_{12} & \cdots & g_{1 r} \\
g_{21} & g_{22} & \cdots & g_{2 r} \\
& & \vdots & \\
g_{n 1} & g_{n 2} & \cdots & g_{n r}
\end{array}\right], \\
\boldsymbol{G}_{d k}=\operatorname{diag}\left[\begin{array}{llll}
g_{1 k} & g_{2 k} & \cdots & g_{n k}
\end{array}\right]=\operatorname{diag}\left[\left\{g_{l k}\right\}_{l=1, \ldots, n}\right],
\end{gathered}
$$

while $\boldsymbol{G}_{d k} \in \mathfrak{R}_{+}^{n \times n}$.

When the above conditions hold, the control gain matrix $\boldsymbol{K}$ is given as

$$
\boldsymbol{K}_{d k}=\boldsymbol{R}_{k} \boldsymbol{P}^{-1}, \quad \boldsymbol{k}_{k}^{T}=\boldsymbol{l}^{T} \boldsymbol{K}_{d k}, \quad \boldsymbol{K}=\left[\begin{array}{c}
\boldsymbol{k}_{1}^{T} \\
\vdots \\
\boldsymbol{k}_{r}^{T}
\end{array}\right]
$$

where $\boldsymbol{K} \in \mathfrak{R}_{+}^{r \times n}$. 
Proof In analogy with (30), (31) it is evident that $\boldsymbol{F}_{c}$ be a strictly positive matrix if all its elements satisfy the conditions

$$
f_{j l}-\sum_{k=1}^{r} g_{k j} k_{k l}>0 \text { for all } j, l=1,2, \ldots, n .
$$

Thus, denoting,

$$
\boldsymbol{K}_{d k}=\operatorname{diag}\left[\begin{array}{llll}
k_{k 1} & k_{k 2} & \cdots & k_{k n}
\end{array}\right]=\left[\left\{k_{k j}\right\}_{j=1, \ldots, n}\right], k=1,2 \ldots, r,
$$

in the same way as above then (86) can be interpreted for $h=0,1,2, \ldots \mathrm{n}-1$ as

$$
\boldsymbol{T}^{h} \boldsymbol{F}(j, j+h)_{(1 \leftrightarrow n) / n} \boldsymbol{T}^{h T}-\sum_{k=1}^{r} \boldsymbol{T}^{h} \boldsymbol{G}_{d k} \boldsymbol{T}^{h T} \boldsymbol{K}_{d k} \succ 0 .
$$

Since multiplying the right side of (88) by $\boldsymbol{P}$ leads to

$$
\boldsymbol{T}^{h} \boldsymbol{F}(j, j+h)_{(1 \leftrightarrow n) / n} \boldsymbol{T}^{h T} \boldsymbol{P}-\sum_{k=1}^{r} \boldsymbol{T}^{h} \boldsymbol{G}_{d k} \boldsymbol{T}^{h T} \boldsymbol{K}_{d k} \boldsymbol{P} \succ 0,
$$

with the notation

$$
\boldsymbol{R}_{k}=\boldsymbol{K}_{d k} \boldsymbol{P}
$$

then (89) implies (78).

Inserting the closed-loop system matrix (70) into (5) gives

$$
\left[\begin{array}{cc}
-\boldsymbol{P}+\boldsymbol{Q} & \boldsymbol{P}\left(\boldsymbol{F}-\sum_{k=1}^{r} \boldsymbol{g}_{k} \boldsymbol{k}_{k}^{T}\right)^{T} \\
\left(\boldsymbol{F}-\sum_{k=1}^{r} \boldsymbol{g}_{k} \boldsymbol{k}_{k}^{T}\right) \boldsymbol{P} & -\boldsymbol{P}
\end{array}\right] \prec 0,
$$

and using from (90) implying notation

$$
\boldsymbol{r}_{k}^{T}=\boldsymbol{k}_{k}^{T} \boldsymbol{P}
$$

(91) implies (77). This concludes the proof.

Theorem 6 The Luenberger observer (71), (72) is stable strictly positive if the system (1), (2) is strictly positive and there exists a positive definite diagonal matrices $\boldsymbol{U}, \boldsymbol{V}, \boldsymbol{W}_{k} \in \mathfrak{R}^{n \times n}$ such that for $h=0,1,2, \ldots n-1, k=1,2, \ldots m$,

$$
\begin{gathered}
{\left[\begin{array}{cc}
-\boldsymbol{V}+\boldsymbol{U} & \boldsymbol{F}^{T} \boldsymbol{V}-\sum_{k=1}^{m} \boldsymbol{c}_{k} \boldsymbol{w}_{k} \\
* & -\boldsymbol{V}
\end{array}\right] \prec 0} \\
\frac{1}{2}\left(\boldsymbol{V} \boldsymbol{T}^{h} \boldsymbol{F}(i+h, i)_{(1 \leftrightarrow n) / n} \boldsymbol{T}^{h T}-\sum_{k=1}^{m} \boldsymbol{W}_{k} \boldsymbol{T}^{h} \boldsymbol{C}_{d k} \boldsymbol{T}^{h T}\right)+(*) \succ 0,
\end{gathered}
$$




$$
\begin{gathered}
\boldsymbol{V}=\operatorname{diag}\left[\begin{array}{llll}
v_{1} & v_{2} & \cdots & v_{n}
\end{array}\right] \succ 0, \\
\boldsymbol{U}=\operatorname{diag}\left[\begin{array}{llll}
u_{1} & u_{2} & \cdots & u_{n}
\end{array}\right] \succ 0, \\
\boldsymbol{W}_{k}=\operatorname{diag}\left[\begin{array}{llll}
w_{k 1} & w_{k 2} & \cdots & w_{k n}
\end{array}\right] \succ 0, \\
\boldsymbol{w}_{k}^{T}=\left[\begin{array}{lllll}
w_{k 1} & w_{k 2} & \cdots & w_{k n}
\end{array}\right]=\boldsymbol{l}^{T} \boldsymbol{W}_{k}, \quad \boldsymbol{l}=\left[\begin{array}{llll}
1 & 1 & \cdots & 1
\end{array}\right]^{T},
\end{gathered}
$$

where $\boldsymbol{T}$ is defined in (24), $\boldsymbol{A}(i+h, i)_{(1 \leftrightarrow n) / n}$ is introduced in (53),

$$
\begin{aligned}
& \boldsymbol{C}=\left[\begin{array}{cccc}
c_{11} & c_{12} & \cdots & c_{1 n} \\
c_{21} & c_{22} & \cdots & c_{2 n} \\
& & \vdots & \\
c_{m 1} & c_{m 2} & \cdots & c_{m n}
\end{array}\right] \\
& \boldsymbol{C}_{d k}=\operatorname{diag}\left[\begin{array}{llll}
c_{k 1} & c_{k 2} & \cdots & c_{k n}
\end{array}\right]=\operatorname{diag}\left[\left\{c_{k l}\right\}_{l=1, \ldots, n}\right],, k=1,2 \ldots, m,
\end{aligned}
$$

while $\boldsymbol{C}_{d k} \in \mathfrak{R}^{n \times n}$.

When the above conditions hold, the observer gain matrix $\boldsymbol{J}$ is given as

$$
\boldsymbol{J}_{d k}=\boldsymbol{V}^{-1} \boldsymbol{W}_{k}, \quad \boldsymbol{j}_{k}=\boldsymbol{J}_{d k} \boldsymbol{l}, \quad \boldsymbol{J}=\left[\begin{array}{lll}
\boldsymbol{j}_{1} & \cdots & \boldsymbol{j}_{m}
\end{array}\right]
$$

where $\boldsymbol{J} \in \mathfrak{R}_{+}^{n \times m}$.

Proof In analogy with (55), (55) it is evident that $\boldsymbol{F}_{e}$ be a positive matrix if

$$
f_{l j}-\sum_{k=1}^{m} j_{k l} c_{k j}>0 \text { for all } l, j=1,2, \ldots, n .
$$

Thus, denoting,

$$
\boldsymbol{J}_{d k}=\operatorname{diag}\left[\begin{array}{llll}
j_{1 k} & j_{2 k} & \cdots & j_{n k}
\end{array}\right]=\left[\left\{j_{l k}\right\}_{l=1, \ldots, n}\right], k=1,2 \ldots, m,
$$

and, using the above notations then (102) implies for $h=0,1,2, \ldots n-1$ that

$$
\boldsymbol{T}^{h} \boldsymbol{F}(i, i+h)_{(1 \leftrightarrow n) / n} \boldsymbol{T}^{h T}-\sum_{k=1}^{m} \boldsymbol{J}_{d k} \boldsymbol{T}^{h} \boldsymbol{C}_{d k} \boldsymbol{T}^{h T} \succ 0 .
$$

Since multiplying the left side of (104) by $V$ leads to

$$
\boldsymbol{V} \boldsymbol{T}^{h} \boldsymbol{F}(i, i+h)_{(1 \leftrightarrow n) / n} \boldsymbol{T}^{h T}-\sum_{k=1}^{m} \boldsymbol{V} \boldsymbol{J}_{d k} \boldsymbol{T}^{h} \boldsymbol{C}_{d k} \boldsymbol{T}^{h T} \succ 0,
$$

with the notation

$$
\boldsymbol{W}_{k}=\boldsymbol{V} \boldsymbol{J}_{d k}
$$

then (105) implies (94). 
Inserting the observer system matrix (75) into (6) gives

$$
\left[\begin{array}{cc}
-\boldsymbol{V}+\boldsymbol{U} & \left(\boldsymbol{F}-\sum_{k=1}^{m} \boldsymbol{j}_{k} \boldsymbol{c}_{k}^{T}\right)^{T} \boldsymbol{V} \\
\boldsymbol{V}\left(\boldsymbol{F}-\sum_{k=1}^{m} \boldsymbol{j}_{k} \boldsymbol{c}_{k}^{T}\right) & -\boldsymbol{V}
\end{array}\right] \prec 0
$$

and using the notation

$$
\boldsymbol{w}_{k}=\boldsymbol{V} \boldsymbol{j}_{k}, \quad \boldsymbol{w}_{k}^{T}=\boldsymbol{j}_{k}^{T} \boldsymbol{V},
$$

then (107) implies (93). This concludes the proof.

\section{Illustrative example}

The generating strictly Metzlerian system is represented by the continuous-time linear state-space model with the parameters

$$
\begin{gathered}
\boldsymbol{A}=\left[\begin{array}{rrrr}
-3.3800 & 0.2080 & 6.7150 & 5.6760 \\
0.5810 & -4.2900 & 0.0100 & 0.6750 \\
1.0670 & 4.2730 & -6.6540 & 5.8930 \\
0.0480 & 4.2730 & 1.3430 & -2.1040
\end{array}\right], \quad \boldsymbol{B}=\left[\begin{array}{lll}
0.4000 & 0.1888 \\
0.5679 & 0.2030 \\
0.1136 & 0.3146 \\
0.1136 & 0.1701
\end{array}\right] \\
\boldsymbol{C}=\left[\begin{array}{llll}
4 & 0 & 1 & 0 \\
0 & 0 & 0 & 1
\end{array}\right]
\end{gathered}
$$

It is possible to verify that the Metzler matrix $\boldsymbol{A}$ is instable with the eigenvalue spectrum

$$
\rho(\boldsymbol{A})=\{1.5599, \quad-8.7316, \quad-4.6282 \pm 1.7583 \mathrm{i}\} .
$$

Converting for the sampling period $t_{s}=0.02 \mathrm{~s}$, the discrete-time system parameters are

$$
\boldsymbol{F}=\left[\begin{array}{llll}
0.9361 & 0.0139 & 0.1230 & 0.1150 \\
0.0108 & 0.9184 & 0.0011 & 0.0133 \\
0.0198 & 0.0814 & 0.8782 & 0.1098 \\
0.0016 & 0.0813 & 0.0247 & 0.9609
\end{array}\right], \quad \boldsymbol{G}=\left[\begin{array}{ll}
0.0081 & 0.0043 \\
0.0109 & 0.0039 \\
0.0028 & 0.0063 \\
0.0027 & 0.0036
\end{array}\right]
$$

where

$$
\rho(\boldsymbol{F})=\left\{\begin{array}{lll}
1.0317, & 0.8398, & 0.9110 \pm 0.0321 \mathrm{i}
\end{array}\right\}
$$

is the unstable eigenvalue spectrum of $\boldsymbol{F}$.

To solve the stabilization task, the auxiliary parameters are constructed as

$$
\boldsymbol{T}=\left[\begin{array}{llll}
0 & 0 & 0 & 1 \\
1 & 0 & 0 & 0 \\
0 & 1 & 0 & 0 \\
0 & 0 & 1 & 0
\end{array}\right]
$$




$$
\begin{aligned}
\boldsymbol{F}(i, i)_{(1 \leftrightarrow 4)}= & {\left[\begin{array}{llll}
0.9361 & & & \\
& 0.9184 & & \\
& & 0.8782 & \\
& & & 0.9609
\end{array}\right] } \\
\boldsymbol{F}(i, i+1)_{(1 \leftrightarrow 4) / 4}= & {\left[\begin{array}{llll}
0.0139 & & & \\
& 0.0011 & & \\
& & 0.1098 & \\
& & & 0.0016
\end{array}\right], } \\
\boldsymbol{F}(i, i+2)_{(1 \leftrightarrow 4) / 4}= & {\left[\begin{array}{lllll}
0.1230 & & & \\
& 0.0133 & & \\
& & 0.0198 & \\
& & & 0.0813
\end{array}\right], } \\
\boldsymbol{F}(i, i+3)_{(1 \leftrightarrow 4) / 4}= & {\left[\begin{array}{llll}
0.1150 & & & \\
& 0.0108 & 0.0814 & \\
& & & 0.0247
\end{array}\right] . }
\end{aligned}
$$

Using the SeDuMi package [28] to solve the given set of LMIs (77)-(83) in MAT$\mathrm{LAB}$ environment the $\mathrm{LMI}$ variables are

$$
\begin{gathered}
\boldsymbol{P}=\operatorname{diag}\left[\begin{array}{llll}
0.5779 & 0.0082 & 0.1425 & 0.0417
\end{array}\right], \\
\boldsymbol{Q}=\operatorname{diag}\left[\begin{array}{llll}
0.8228 & 0.0121 & 0.1168 & 0.0144
\end{array}\right] * 10^{-3}, \\
\boldsymbol{R}_{1}=\operatorname{diag}\left[\begin{array}{llll}
0.3403 & 0.0018 & 0.0016 & 0.0005
\end{array}\right], \\
\boldsymbol{R}_{2}=\operatorname{diag}\left[\begin{array}{llll}
0.0040 & 0.0226 & 0.0322 & 0.1391
\end{array}\right] .
\end{gathered}
$$

The control law gain matrix $K \in \mathfrak{R}^{2 \times 4}$, computed by using (84), is positive matrix, since

$$
\begin{aligned}
\boldsymbol{k}_{1}^{T} & =\left[\begin{array}{llll}
0.5889 & 0.2178 & 0.0109 & 0.0127
\end{array}\right], \\
\boldsymbol{k}_{2}^{T} & =\left[\begin{array}{llll}
0.0069 & 2.7546 & 0.2259 & 3.3340
\end{array}\right], \\
\boldsymbol{K} & =\left[\begin{array}{llll}
0.5889 & 0.2178 & 0.0109 & 0.0127 \\
0.0069 & 2.7546 & 0.2259 & 3.3340
\end{array}\right],
\end{aligned}
$$

which implies the stable strictly positive matrix of closed-loop system matrix

$$
\boldsymbol{F}_{c}=\left[\begin{array}{llll}
0.9313 & 0.0004 & 0.1220 & 0.1006 \\
0.0043 & 0.9052 & 0.0001 & 0.0001 \\
0.0182 & 0.0634 & 0.8767 & 0.0888 \\
0.0001 & 0.0708 & 0.0239 & 0.9489
\end{array}\right],
$$




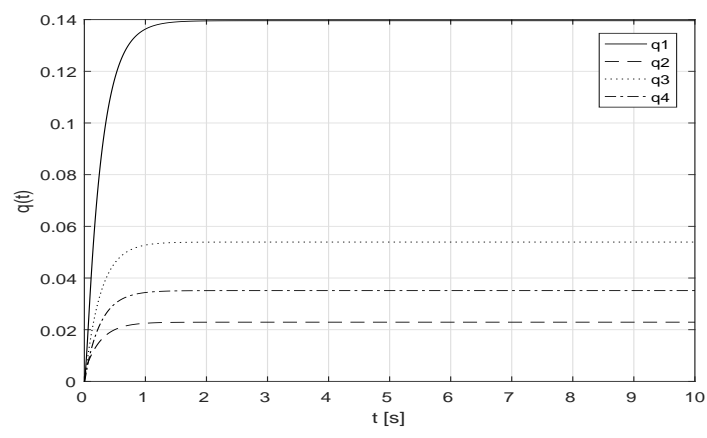

Figure 1: State variables response

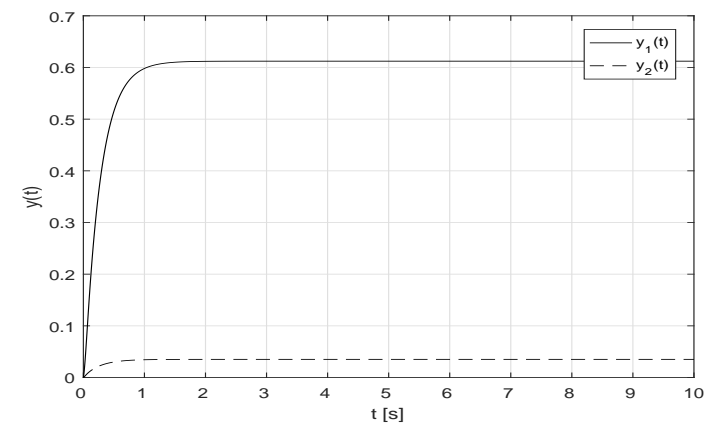

Figure 2: Output variables response

with the eigenvalue spectrum

$$
\rho\left(\boldsymbol{F}_{c}\right)=\{0.8390, \quad 0.9979, \quad 0.9126 \pm 0.0222 \mathrm{i}\} .
$$

Analyzing the numerical results, it is evident that the stable strictly positive matrix $\boldsymbol{F}_{c}$ is diagonally dominant.

The obtained results are illustrated in Fig. 1 and Fig. 2, where the state variables vector $\boldsymbol{q}(t)$ as well as the output variables vector $\boldsymbol{y}(t)$ are positive when the input in the closed-loop system is the positive vector $\boldsymbol{w}^{T}=\left[\begin{array}{ll}0.44 & 0.20\end{array}\right]$ and

$$
\boldsymbol{u}(i)=-\boldsymbol{K} \boldsymbol{q}(i)+\boldsymbol{w} .
$$

To design the strictly positive observer, the auxiliary parameters are constructed so that

$$
\boldsymbol{F}(i+1, i)_{(1 \leftrightarrow 4) / 4}=\left[\begin{array}{cccc}
0.0108 & & & \\
& 0.0814 & & \\
& & 0.0247 & \\
& & & 0.1150
\end{array}\right],
$$




$$
\begin{aligned}
\boldsymbol{F}(i+2, i)_{(1 \leftrightarrow 4) / 4} & =\left[\begin{array}{llll}
0.0198 & & & \\
& 0.0813 & & \\
& & 0.1230 & \\
& & & 0.0133
\end{array}\right], \\
\boldsymbol{F}(i+3, i)_{(1 \leftrightarrow 4) / 4} & =\left[\begin{array}{llll}
0.0016 & & & \\
& 0.0139 & & \\
& & 0.0011 &
\end{array}\right] .
\end{aligned}
$$

Therefore, the set of LMIs (93)-(98) is satisfied for the LMI variables

$$
\begin{aligned}
\boldsymbol{V} & =\operatorname{diag}\left[\begin{array}{llll}
2.1809 & 2.3636 & 1.9798 & 1.9845
\end{array}\right], \\
\boldsymbol{U} & =\operatorname{diag}\left[\begin{array}{llll}
0.6628 & 0.1221 & 0.1706 & 0.7234
\end{array}\right], \\
\boldsymbol{W}_{1} & =\operatorname{diag}\left[\begin{array}{llll}
0.2016 & 0.0013 & 0.0046 & 0.0004
\end{array}\right], \\
\boldsymbol{W}_{2} & =\operatorname{diag}\left[\begin{array}{llll}
0.1277 & 0.0159 & 0.1201 & 1.2188
\end{array}\right] .
\end{aligned}
$$

The observer gain matrix $\boldsymbol{J} \in \mathfrak{R}_{+}^{4 \times 2}$ is computed by using (101) as

$$
\boldsymbol{j}_{1}=\left[\begin{array}{l}
0.0924 \\
0.0005 \\
0.0023 \\
0.0002
\end{array}\right], \quad \boldsymbol{j}_{2}=\left[\begin{array}{l}
0.0585 \\
0.0067 \\
0.0607 \\
0.6142
\end{array}\right], \quad \boldsymbol{J}=\left[\begin{array}{ll}
0.0924 & 0.0585 \\
0.0005 & 0.0067 \\
0.0023 & 0.0607 \\
0.0002 & 0.6142
\end{array}\right],
$$

which leads to the stable strictly positive matrix of the observer dynamics

$$
\boldsymbol{F}_{e}=\left[\begin{array}{llll}
0.5888 & 0.0141 & 0.0292 & 0.0574 \\
0.0088 & 0.9184 & 0.0005 & 0.0066 \\
0.0106 & 0.0814 & 0.8758 & 0.0492 \\
0.0008 & 0.0813 & 0.0245 & 0.3463
\end{array}\right]
$$

with the stable eigenvalue spectrum

$$
\rho\left(\boldsymbol{F}_{e}\right)=\{0.3438, \quad 0.5647, \quad 0.8757,0.9231\} .
$$

Analyzing the results, it is evident that the stable positive observer is designed to the unstable positive system. Also in this case the stable strictly positive matrix $\boldsymbol{F}_{e}$ is diagonally dominant and, consequently, the observer state response is aperiodic and positive.

With respect to the $n \times r$ boundary conditions (78) it is evident that not every linear positive system is stabilizable by the state control, while it is clear that a linear discretetime system is also stabilizable when the generating linear continuous-time system is stabilizable. Using more than a hundred randomly generated Metzlerian continuous-time linear systems, it was discovered that only unstable systems with dominant diagonal elements of the Metzler matrix $\boldsymbol{A}$ are potentially stabilizable. To illustrate this interpretation it is possible to verify that for the system used in the presented example the LMIs are close to singular if there is the element $\boldsymbol{A}(1,1)=a_{11}>-0.5$, which leads to an unstable solution. 


\section{Concluding remarks}

A novel approach is presented in the paper to address the problem of effectively computing a state feedback control law gain that makes the positive system in closed-loop to be strictly positive and stable, as well as of recounting the observer gain that establish the stable strictly positive structures of Luenberger observers. Based on a stable strictly positive matrix properties, algebraic constraints implying from linear programming approach are reformulated as a set of LMIs, and replenished by the Lyapunov matrix inequality in the sense of the second Lyapunov method. It is derived that all matrix variables associated with this LMIs ensemble have to be positive definite and diagonal. The proposed approach provides a numerically reliable computational framework, as illustrated using a numerical example, and might be extended to other particular cases.

Further research topics include the problems of forced mode in positive systems as well as nonlinear positive systems.

\section{References}

[1] F. Cacace, L. Farina, R. Setola and A. Germani (Eds.): Positive Systems. Lecture Notes in Control and Information Sciences LNCIS, 471 Springer, Cham, 2016.

[2] J.M. Carnicer, J.M. Pena and R.A. Zalik: Strictly totally positive systems. J. Approximation Theory, 92 (1998), 411-441.

[3] T. KaczoreK: Positive $1 D$ and 2D Systems. Springer-Verlag, London, 2002.

[4] T. Kaczorek and L. Sajewski: The Realization Problem for Positive and Fractional Systems. Springer, Cham, 2014.

[5] Z. Shu, J. LAM, H. GaO, B. Du and L. Wu: Positive observers and dynamic output-feedback controllers for interval positive linear systems. IEEE Tran. Circuits and Systems, 55(10), (2008), 3209-3222.

[6] N.K. SON and D. HinRICHSEN: Robust stability of positive continuous time systems. Numerical Functional Analysis and Optimization, 17(5-6), (1996), 649-659.

[7] J. BACK and A. Astolfi: Design of positive linear observers for positive linear systems via coordinate transformations and positive realizations. SIAM J. on Control and Optimization, 47(1), (2008), 345-373.

[8] O. Pastravanu and M.H. Matcovschi: $(M, \beta)$-stability of positive linear systems. Mathematical Problems in Engineering, Article ID 9605464, 11p, (2016).

[9] T. Kaczorek: Positive stable realizations with system Metzler matrices. Archives of Control Sciences, 21(2), (2011), 122-142. 
[10] B. Canto, R. Canto and S. Kostova: Stabilization of positive linear discretetime systems by using a Brauers theorem. Scientific World Journal, Article ID 856356, 6p, (2014).

[11] T. KACZOREK: Determination of positive realizations with reduced numbers of delays or without delays for discrete-time linear systems. Archives of Control Sciences, 22(4), (2012), 451-465.

[12] X. XUE and L. GuO: A kind of nonnegative matrices and its application on the stability of discrete dynamical systems. J. Mathematical Analysis and Application, 331(2), (2007), 1113-1121.

[13] H. GAO, J. LAM, C. WANG and S. XU: Control for stability and positivity. Equivalent conditions and computation. IEEE Trans. Automatic Control, 52(9), (2005), 540-544.

[14] M. AIt RAMI and F. TADEO: Controller synthesis for positive linear systems with bounded controls. IEEE Trans. Circuits and Systems, 54(2), (2007), 151-155.

[15] A. Berman, M. Neumann and R. Stern: Nonnegative Matrices in Dynamic Systems. John Wiley \& Sons, New York, 1989.

[16] D.G. Luenberger: Introduction to Dynamic Systems. Theory, Models and Applications. John Wiley \& Sons, New York, 1979.

[17] M. Ait Rami and F. TAdeO: Positive observation problem for linear discrete positive systems. Proc. 45th IEEE Conf. on Decision \& Control, San Diego, CA, USA, (2006), 4729-4733.

[18] B. Shafai and A. Oghbaee: Positive quadratic stabilization of uncertain linear system. Proc. 2014 IEEE Multi-conference on Systems and Control, Antibes, France, (2014), 1412-1417.

[19] C.L. Philips and H.T. Nagle: Digital Control System Analysis and Design. Prentice Hall, Englewood Cliffs, 1984.

[20] P. De LeEnheER and D. Aeyels: Stabilization of positive linear systems. Systems \& Control Letters, 44(4), (2001), 259-271.

[21] L. FARINA and S. RinAldi: Positive Linear Systems. Theory and Applications. John Wiley \& Sons, New York, 2000.

[22] C.A.R. Crusius and A. TrofinO: Sufficient LMI conditions for output feedback control problems. IEEE Trans. Automatic Control, 44(5), (1999), 1053-1057.

[23] A. Filasová, D. Gontkovič and D. Krokavec: Observer-based fault estimation for linear systems with distributed time delay. Archives of Control Sciences, 23(2), (2013), 169-186. 
[24] G. Birkhoff and S. Mac Lane: A survey of Modern Algebra. Macmillan Publishing, New York, 1977.

[25] D.J.S. Robinson: An Introduction to Abstract Algebra. Walter de Gruyter, Berlin, 2003.

[26] R.A. Horn and C.R. Johnson: Matrix Analysis. Cambridge University Press, New York, 2013.

[27] W.M. Haddad and V. Chellaboina: Nonlinear Dynamical Systems and Control. A Lyapunov-Based Approach. Princeton Univiversity Press, Princeton, 2008.

[28] D. Peaucelle, D. Henrion, Y. Labit and K. Taitz: User's Guide for SeDuMi Interface. LAAS-CNRS, Toulouse, 2002. 